\title{
Direct Adaptive Current Control in Space Vector Overmodulation Mode
}

\author{
Andreas Liske, Michael Braun Karlsruhe Institute of Technology (KIT) \\ Elektrotechnisches Institut (ETI) - Electrical Drives and Power Electronics \\ Kaiserstr. 12, 76131 Karlsruhe, Germany \\ Andreas.Liske@kit.edu,www.eti.kit.edu
}

\begin{abstract}
In this paper an algorithm is presented how the current slopes of the freewheeling switching states of an VSIdriven 3-phase isotropic synchronous machine can be obtained by only evaluating the measured current slopes during the two applied active switching states. This enables the identification of the freewheeling current slopes in operating conditions where the freewheeling switching states are too short for a direct measurement. Notably, the use of the "Direct Adaptive Current Control" in the overmodulation range is made possible with the proposed technique.
\end{abstract}

\section{INTRODUCTION}

In recent years many advanced current control strategies have been investigated and published. Besides excellent control quality and high dynamics, the goals are an ideal setting of the control parameters, increasing robustness, to be able to replace the mechanical speed and torque sensors or simply to reduce the time and effort during initial implementation of a new drive system setup [1]. Especially model predictive control (MPC) [2] offers great possibilities since even complex physical sideeffects that are often neglected can be controlled. This is done by online calculation of a detailed model, that includes for example saturation effects [3] or even crosscoupling effects [4] of permanent magnet synchronous machines. The drawbacks of many predictive control schemes on the other hand are versatile and often combined. One of them is the dependency on control path parameters that may vary during operation, leading to a suboptimal and sometimes fragile control setup. Another is the use of complex mathematical models, which increases the required computing time and can collide with the hard real time demands of a highdynamic drive control system.

In contrary to model predictive control, "Direct Adaptive Current Control" (DACC) is a one-step predictive current control scheme that predicts the behavior of the controlled machine currents without a model, only by evaluating the current slopes. It was first presented in [5] for the control of a DC/DC-converter and for single phase applications [6]. Later it was adapted for the current control of magnetic isotropic 3-phase synchronous machines (with $L_{\mathrm{d}}=L_{\mathrm{q}}$ ) [7], magnetic anisotropic synchronous machines $\left(L_{\mathrm{d}} \neq L_{\mathrm{q}}\right)$ [8] and for 3-phase induction motors [9] as well. It shows excellent control quality and high dynamics. At the same time it does not need any control path parameters, no complex model, no test pulses, no offline calculations or cost functions and the computational effort is comparatively little.

It is based on the detection of the current slopes during each switching state of the utilized inverter within one period. To enable the measurement of the current slopes during each switching state, a certain minimum on-time of the switching states is necessary. In normal SPVM operating mode this is usually no problem, since every relevant switching state is applied more or less regularly. But in overmodulation mode, the zero switching state is never applied [10] and the current slopes during freewheeling condition can not be measured. However, the knowledge of them is essential for the DACCcontrol algorithm. For this reason a defined minimum on-time for the zero switching state of about $10 \%$ of the duty cycle was suggested and implemented in previous publications. This indeed solves the problem but leads to a significantly reduced modulation index.

In this paper a new algorithm is presented, how the current slopes of the freewheeling switching states can be obtained only by evaluating the current slopes of the active switching states. The zero switching states are not necessary at all, which enables an implementation of the DACC without a minimum on-time of the zero switching state. Hence the use of it in the overmodulation range is made possible.

After a brief introduction to the DACC control scheme in section II, the new algorithm to identify the current slopes of the freewheeling switching state is developed and presented in section III. In section IV simulation results are presented. 


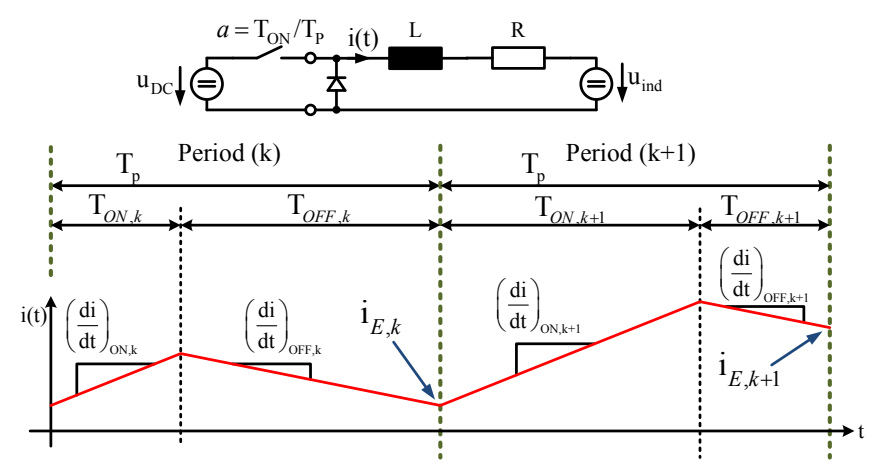

Figure 1. Basic working principle of the DACC, illustrated with a one-phase RL-load

\section{Working PRINCIPLE OF THE DiRECT AdAPTIVE CURRENT CONTROL}

\section{A. DACC with a one-phase RL-load}

The basic working principle of the DACC control scheme is best explained with a one-phase RL-load as depicted in Figure 1. By operating the switch with the duty cycle $a=T_{\mathrm{ON}} / T_{\mathrm{P}}$, the voltage $U_{\mathrm{DC}}$ is applied to the RL-load. Given that the switching frequency is high enough, the current will rise and fall linearly in straight line segments. The current slopes for each switching state of period $\mathrm{k}$, which is $(\mathrm{di} / \mathrm{dt})_{\mathrm{ON}, k}$ and $(\mathrm{di} / \mathrm{dt})_{\mathrm{OFF}, k}$ can be assumed to be the same in period $k+1$ if the applied voltage, the inductance and the resistance are approximately constant for two consecutive periods. This usually is given due to the relatively high switching frequency.

Once the current slopes of period $\mathrm{k}$ are known for every switching state, the necessary duty cycle for a given current setpoint $\mathrm{i}_{\mathrm{E}, k+1}$ that should be reached at the end of the next period $\mathrm{k}+1$ can be calculated easily with a linear equation. The knowledge of the absolute voltage, the inductance and the resistance is not needed.

\section{B. DACC in three-phase applications}

For three phase applications the very same principle can be applied as shown in [6]. Again, the basis for the DACC [8] and the preceding control schemes [6], [7], [11] is the fast detection of the stator current slopes during each switching state $S_{n}$ with $n \in\{1 . .8\}$ of the utilized voltage source inverter (VSI). This can be done in many different ways, such as sensing the current slopes directly with analog differentiator circuits [12], fast oversampling of the current and implementing a least-squares-estimator algorithm to filter the noise [6], [8], [9] or newer undersampling methods [13].
However, the current slopes $\frac{d}{d t} \mathrm{i}_{\mathrm{a}, n, k}$ and $\frac{d}{d t} \mathrm{i}_{\mathrm{f}, k}$ of period $\mathrm{k}$ are used to define the so called "current gradient vectors" $\Delta \mathrm{i}_{\mathrm{a}, n, k}$ and $\Delta \mathrm{i}_{\mathrm{f}, k}[6]$ :

$$
\begin{aligned}
\Delta \underline{\mathrm{i}}_{\mathrm{f}, k} & =\frac{d}{d t} \mathrm{i}_{\mathrm{f}, k} \cdot T_{\mathrm{p}} \\
\Delta \mathrm{i}_{\mathrm{a}, n, k} & =\left(\frac{d}{d t} \mathrm{i}_{\mathrm{a}, n, k}-\frac{d}{d t} \mathrm{i}_{\mathrm{f}, k}\right) \cdot T_{\mathrm{p}}
\end{aligned}
$$

The index a stands for an active switching state, where the machine is connected to the DC link voltage. The index $\mathrm{f}$ indicates the freewheeling switching states respectively. The index $\mathrm{n}$ denotes which one of the six possible switching states $\underline{S}_{1}$ to $\underline{S}_{6}$ is applied. The index $\mathrm{k}$ is the period-counter.

The current gradient vectors $\Delta \dot{i}_{a, n, k}$ describe the current variation that would occur, if only the voltage corresponding to the active switching state with the index $\mathrm{n}$ would be applied to the machine for the whole period $\left(T_{\mathrm{p}}\right)$ with the index k. Similarly $\Delta \mathrm{i}_{\mathrm{f}, k}$ depicts the current variation that would occur, if only a freewheeling switching state would be applied to the machine for the whole period $\mathrm{k}$. In three phase systems this can be represented as vectors in the stator-oriented complex $\alpha \beta$-plane [7] (see Figure 2(a)).

This information about the current variation depending on the switching states can be used to calculate the necessary duty cycles for the next period to reach a desired setpoint value directly. The green vector diagram in Figure 2(a) depicts this essential algorithm of the DACC:

The last value of the stator current at the end of the actual period $\mathrm{k}$ is represented by the red current space vector $\underline{\underline{i}}_{e, k}$. The inner voltage of the machine is effective during the whole period in any switching state, so its influence $\Delta \dot{\mathrm{i}}_{\mathrm{f}, k}$ to the current variation can be added to $\underline{\mathrm{i}}_{\mathrm{e}, k}$ directly. The resulting vector $\underline{\mathrm{i}}_{\mathrm{f}, k+1}$ now represents the origin of the hexagon spanned by the current gradient vectors for the active switching states $i_{a, n, k}$. Since the control algorithm in the FPGA is started shortly before the end of the current period $\mathrm{k}$, the necessary value $\underline{\mathrm{i}}_{\mathrm{e}, k}$ for this equation can not be measured, but can be calculated by extrapolation of the just measured current slopes and the knowledge of the applied duty cycles in period k. With $\underline{i}_{f, k+1}$ as starting point, the necessary duty cycles to reach a given current setpoint value $\underline{i}_{e, k+1}$ at the end of the next period $\mathrm{k}+1$ can be obtained by the projection of the vector $\underline{i}_{a, k+1}$ to the adjacent switching state vectors $\underline{S}_{n}$ (see the green vector diagram in Figure 2(a)).

$$
\begin{aligned}
\underline{\mathrm{i}}_{\mathrm{a}, k+1} & =\underline{\mathrm{i}}_{\mathrm{e}, k+1}-\underline{\mathrm{i}}_{\mathrm{f}, k+1}=\underline{\mathrm{i}}_{\mathrm{e}, k+1}-\underline{\mathrm{i}}_{\mathrm{e}, k}-\Delta \underline{\mathrm{i}}_{\mathrm{f}, k} \\
& \stackrel{!}{=} \underline{\mathrm{i}}_{\mathrm{a}, 3, k+1}+\underline{\mathrm{i}}_{\mathrm{a}, 4, k+1}
\end{aligned}
$$

This is done by using the same geometrical computation formulas as with the well known space vector modulation. It is not necessary to know the absolute voltage that is applied to the machine or its inductance and resistance. 


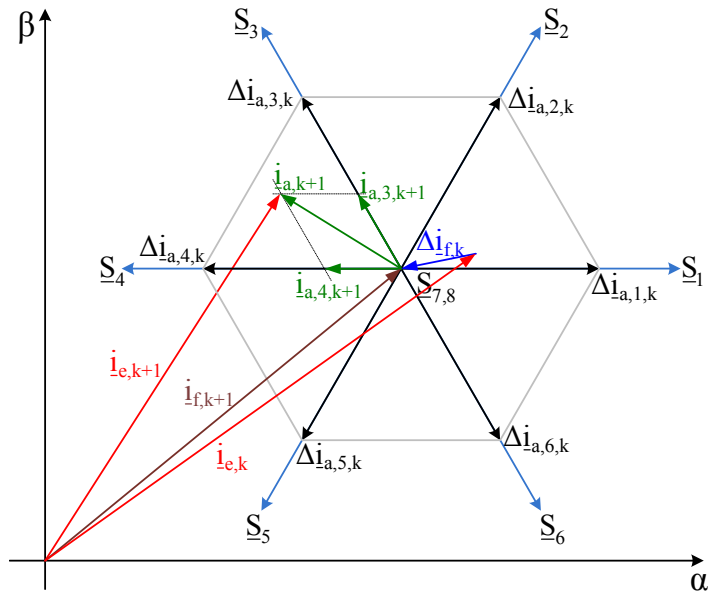

(a) Current gradient vectors $\Delta \underline{\mathbf{i}}_{\mathrm{a}, n, k}$ (black vectors) and $\Delta \underline{\mathrm{i}}_{\mathrm{f}, k}$ (blue vectors), switching state vectors $\underline{S}_{n}$ (blue), area of possible current variation in one pulse period $\mathrm{k}$ (gray hexagon) and the vector diagram of the stator current variation during pulse period $\mathrm{k}+1$ (green)

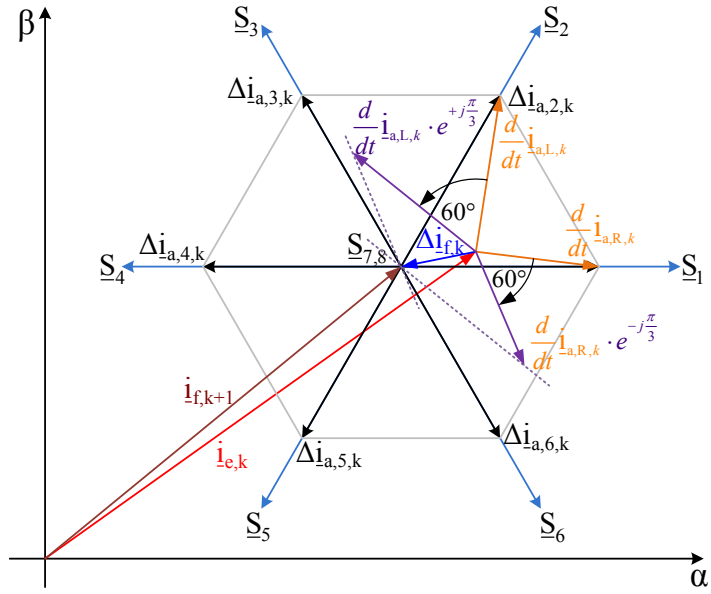

(b) Proposed algorithm to obtain $\Delta \underline{\mathbf{i}}_{\mathrm{f}, k}$ (blue) by rotating the measured current slopes $\frac{d}{d t} \mathrm{i}_{\mathrm{a}, \mathrm{R}, k}$ by $-\frac{\pi}{3}$ and $\frac{d}{d t} \mathrm{i}_{\mathrm{a}, \mathrm{L}, k}$ by $+\frac{\pi}{3}$ (purple) and adding them (dotted purple parallelogram)

Figure 2. Working principle of the Direct Adaptive Current Control 2(a) and the proposed algorithm to obtain $\Delta \mathbf{i}_{f, k}$ by evaluating the measured current slopes of the active switching states 2(b)

The only assumption that has to be valid is, that the current gradient vectors are approximately constant for two consecutive periods, so that $\Delta \mathrm{i}_{\mathrm{a}, n, k} \approx \Delta \mathrm{i}_{\mathrm{a}, n, k+1}$ and $\Delta \mathrm{i}_{\mathrm{f}, k} \approx \Delta \mathrm{i}_{\mathrm{f}, k+1}$ is given.

\section{NEW ALGORITHM TO IDENTIFY THE CURRENT SLOPES OF THE FREEWHEELING SWITCHING STATES IN THE OVERMODULATION RANGE}

\section{A. Derivation of the proposed algorithm}

As can be seen in figure 2(a), the current gradient vectors $\Delta \mathrm{i}_{\mathrm{a}, \mathrm{n}, \mathrm{k}}$ for the active switching states are all of the same length and are located on their corresponding switching state axis $\underline{S}_{n}$. This is valid for magnetic isotropic synchronous machines (with $L_{\mathrm{d}}=L_{\mathrm{q}}$ ) as well as induction motors [7], [9]. Hence, the two adjacent current gradient vectors of one sector just differ by $60^{\circ}$ in their angle (see the black vectors in figure 2(a)). To describe this independent from the absolute switching state, the relative indexes $\mathrm{L}$ for the left and $\mathrm{R}$ for the right active switching state of one sector of the switching state hexagon are introduced.

$$
\Delta \mathrm{i}_{\mathrm{a}, \mathrm{L}, \mathrm{k}}=\Delta \mathrm{i}_{\mathrm{a}, \mathrm{R}, \mathrm{k}} \cdot e^{j \frac{\pi}{3}}
$$

In one period only the two adjacent (left and right) active switching states of the actual sector are used. Their corresponding current slopes $\frac{d}{d t} \mathrm{i}_{\mathrm{a}, \mathrm{L}, k}$ and $\frac{d}{d t} \mathrm{i}_{\mathrm{a}, \mathrm{R}, k}$ are measured and used for the calculation of the left and right current gradient vectors (see equation (2)):

$$
\begin{aligned}
& \Delta \mathrm{i}_{\mathrm{a}, \mathrm{L}, \mathrm{k}}=\left(\frac{d}{d t} \mathrm{i}_{\mathrm{a}, \mathrm{L}, k}-\frac{d}{d t} \mathrm{i}_{\mathrm{f}, k}\right) \cdot T_{\mathrm{p}} \\
& =\frac{d}{d t} \mathrm{i}_{\mathrm{a}, \mathrm{L}, k} \cdot T_{\mathrm{p}}-\Delta \mathrm{i}_{\mathrm{f}, \mathrm{k}} \\
& \Delta \mathrm{i}_{\mathrm{a}, \mathrm{R}, \mathrm{k}}=\left(\frac{d}{d t} \mathrm{i}_{\mathrm{a}, \mathrm{R}, k}-\frac{d}{d t} \mathrm{i}_{\mathrm{f}, k}\right) \cdot T_{\mathrm{p}} \\
& =\frac{d}{d t} \mathrm{i}_{\mathrm{a}, \mathrm{R}, k} \cdot T_{\mathrm{p}}-\Delta \mathrm{i}_{\mathrm{f}, \mathrm{k}}
\end{aligned}
$$

Together with the relation expressed by equation 5 , this is a set of three linear equations with the three unknown variables $\Delta \mathrm{i}_{\mathrm{a}, \mathrm{L}, \mathrm{k}}, \Delta \mathrm{i}_{\mathrm{a}, \mathrm{R}, \mathrm{k}}$ and $\Delta \mathrm{i}_{\mathrm{f}, \mathrm{k}}$ which can be solved: Rearranging equation (6) for $\Delta \mathrm{i}_{\mathrm{f}, \mathrm{k}}$, then substitution of $\Delta \mathrm{i}_{\mathrm{a}, \mathrm{L}, \mathrm{k}}$ with the relation of equation (5) and inserting the result in equation (7) leads to the following calculation rule:

$$
\Delta \mathrm{i}_{\mathrm{f}, \mathrm{k}}=\left(\frac{d}{d t} \mathrm{i}_{\mathrm{a}, \mathrm{L}, k} \cdot e^{+j \frac{\pi}{3}}+\frac{d}{d t} \mathrm{i}_{\mathrm{a}, \mathrm{R}, k} \cdot e^{-j \frac{\pi}{3}}\right) \cdot T_{\mathrm{p}}
$$

The geometrical interpretation of this algorithm is shown in Figure 2(b). The orange vectors are the measured current slopes of the recent period. The left one is rotated by $+60^{\circ}$ and the right one by $-60^{\circ}$. This is drawn as the purple vectors. The vector sum of those purple vectors is illustrated with the purple dotted lines and results in the current gradient vector of the freewheeling switching state $\Delta \mathbf{i}_{\mathrm{f}, \mathrm{k}}$. 


\section{B. Limitations of the proposed algorithm}

The necessary vector rotation by the two constant angles of $\pm 60^{\circ}$ as well as the vector sum can easily be implemented in a control hardware, such as a DSP or FPGA, since the computational effort is very little. The one major necessity for this algorithm though is, that the measurement values of the two current slopes of the adjacent active switching states are valid. This is given, if the on-time of the two active switching states is greater than a certain minimum, and depends on the utilized power semiconductor switching devices, gatedrivers, time requirements, measurement technique and noise. The resulting areas in the space vector hexagon in which the algorithm will work are depicted in figure 3 as the green areas. In the red areas in figure 3, one of the two adjacent active switching states is too short to obtain valid measurement results of the current slopes. Here, the proposed algorithm of equation (8) will not deliver valid results.

To overcome this restriction, the inertial nature of $\Delta \mathrm{i}_{\mathrm{f}}$ comes in handy. Since its angular frequency depends on the mechanical speed of the controlled motor, it can be assumed that it will not change significantly during the time in which the current space vector rotates through the red areas $\left(\frac{d}{d t} \arg \left(\Delta \dot{\mathrm{i}}_{\mathrm{f}}\right) \approx\right.$ const. $)$. The same is also a valid assumption for the length of $\Delta \mathrm{i}_{\mathrm{f}}\left(\frac{d}{d t}\left|\Delta \underline{\mathrm{i}}_{\mathrm{f}}\right| \approx\right.$ const. $)$. With this in mind, $\Delta \mathrm{i}_{\mathrm{f}}$ can be determined in the red areas, by just rotating the last valid value of $\Delta \mathrm{i}_{\mathrm{f}}$ each period by the same angle differential than the one that occured in the green areas. This is a linear extrapolation of the absolute value and the angle of $\Delta \mathbf{i}_{\mathrm{f}}$. As soon as the next operation point in a green area is reached, the value of $\Delta \underline{i}_{f}$ as well as the angle differential can be updated on a measured basis.

If the space vector modulation is not in overmodulation mode, freewheeling switching states applied and are always longer in the corners of the hexagon than on the sides of it. This makes the discussed restriction less critical, since $\Delta \underline{i}_{\mathrm{f}}$ can be measured directly.

\section{Simulation Results}

The presented algorithm has been implemented in a Matlab/Simulink-simulation to develop and proof the theory. An isotropic three-phase permanent magnet synchronous machine was simulated together with an VSI with a PWM switching frequency of $8 \mathrm{kHz}$ and a rotational speed of $1000 \mathrm{rpm}$. The DC-link-voltage was set to $50 \mathrm{~V}$ to test the algorithm at moderate duty cycles as well for the freewheeling as also for the active switching states. The measurement of the current slopes was implemented as fast oversampling with $6 \mathrm{MHz}$ and filtering with a least-squares-estimator. This was done in a very detailed manner even to the point of simulation of the planned FPGA with its clock of $120 \mathrm{MHz}$. This was done,

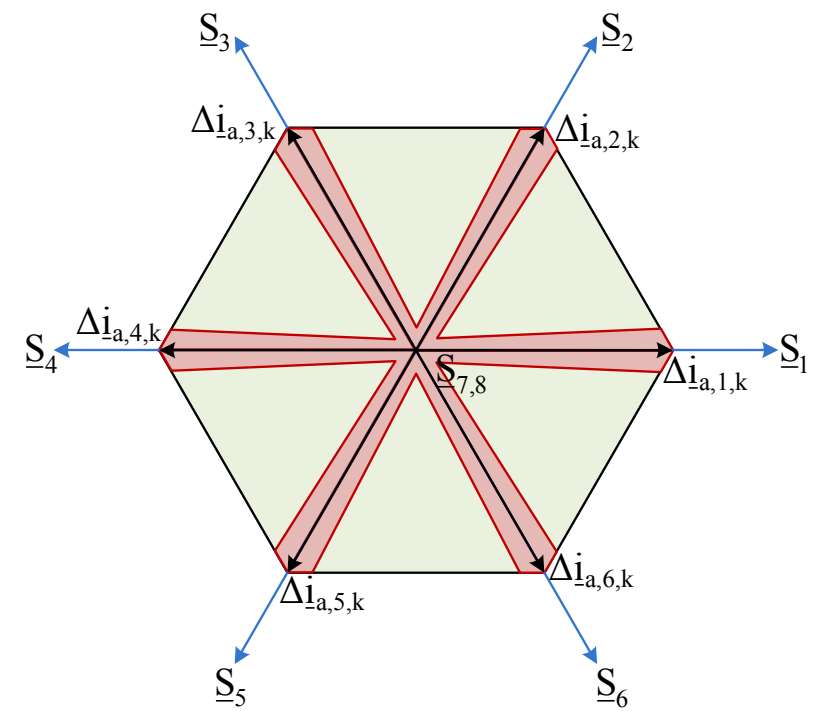

Figure 3. Limitations of the proposed algorithm Green areas: The two adjacent active switching states are both long enough, so that the proposed algorithm delivers good results.

Red areas: not suited for the proposed algorithm without extrapolation (see section III-B)

since the now available simulink-model of the slopedetection shall serve as basis for automated VHDLCode-generation for different target hardware setups in the near future.

The results of this simulation are shown in Figure 4 and in figure 5. The vector diagram in Figure 4 is directly comparable to figure 2(b). However, in figure 4 several periods of the simulation are drawn, whereas in figure 2(b) just the dependencies during one period are shown. The blue vectors, that are arranged around the origin are the ideal values of $\Delta \mathbf{i}_{f}$ and are hardly to see, because the calculated $\Delta \mathbf{i}_{\text {f.calc }}$ are overlaying very close, which approves that the proposed algorithm works. The red and the green vectors are the measured current slopes of the right and the left active switching state $\Delta \underline{i}_{a, R}$ and $\Delta \mathrm{i}_{\mathrm{a}, \mathrm{L}}$. The orange and gray vectors are the rotated current gradient vectors $\Delta \mathrm{i}_{-\mathrm{a}, \mathrm{R}} \cdot e^{-j 60^{\circ}}$ and $\Delta \mathrm{i}_{\mathrm{a}, \mathrm{L}} \cdot e^{+j 60^{\circ}}$. Also a change of the active sector from sector 5 to sector 6 is visible by the marked invalid vectors. During this phase $\Delta \dot{i}_{\text {f,calc,extrapolated }}$ is extrapolated as discussed in section III-B.

In figure 5 the timeline of a complete electrical period is shown. In the top chart, the three motor currents are drawn. The second graph shows the current slopes of the active switching states. It is clearly visible, that they each have three times during this period in which they are zero. This is just the time, when a change of sector takes place, so their on-time is too short to get valid measurement results. In those time slots, the directly calculated $\Delta \mathbf{i}_{\text {f,calc }}$ without any extrapolation, can not deliver valid results. This is obviously visible, 


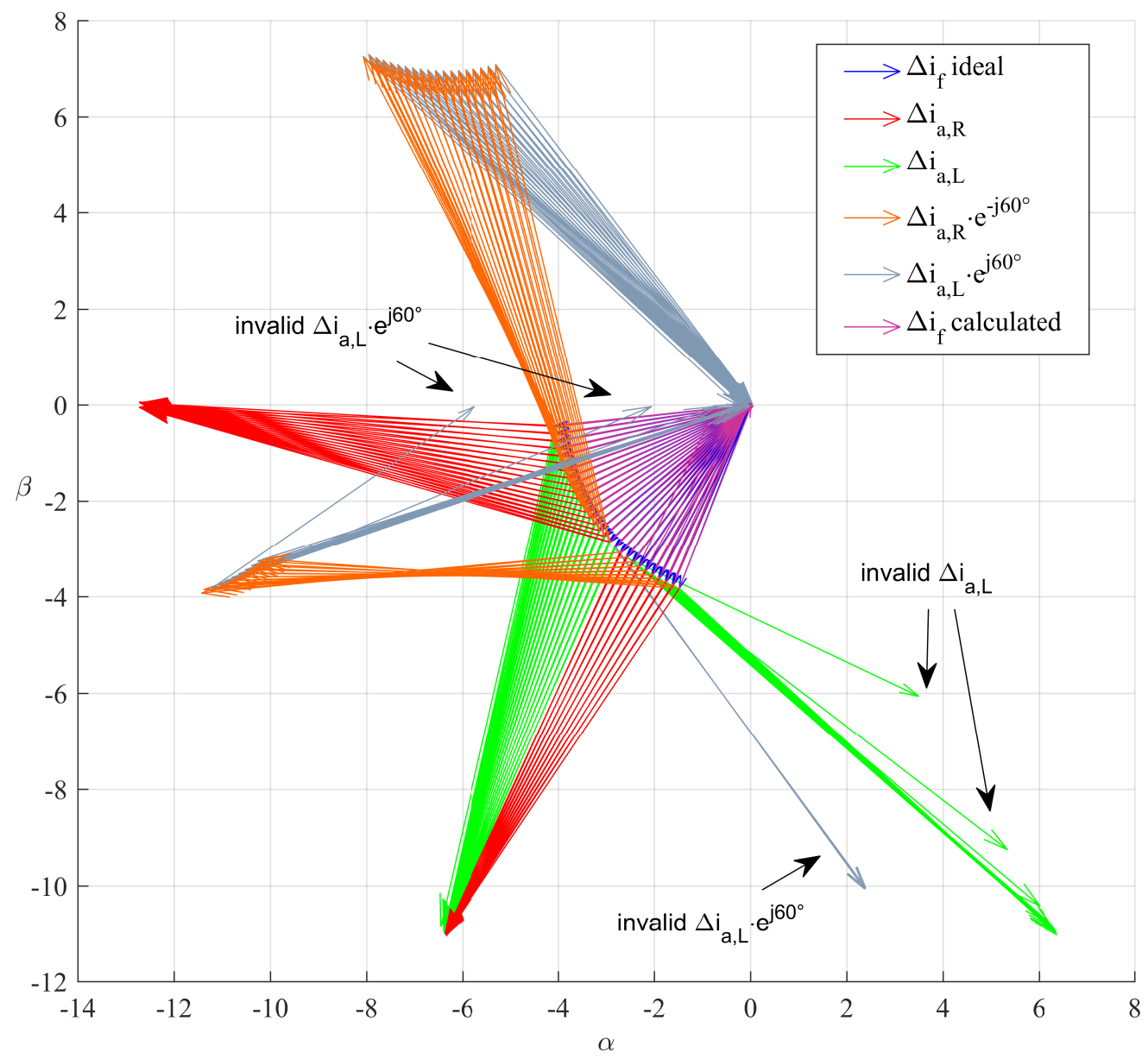

Figure 4. Simulation results of the proposed algorithm as vector diagram (see also figure 2(b))

when looking at the green signals in the two bottom charts: In those, the ideal, the directly calculated and the extrapolated value of $\Delta \mathbf{i}_{\mathrm{f}}$ are compared. The false results of the directly calculated $\Delta \mathbf{i}_{\mathrm{f} \text {,calc }}$ during the time slots in the red areas is obvious.

However, the ideal (blue signal) and the extrapolated (purple and dashed signal) value are almost identical and proof the theory.

\section{CONCLUSION}

This paper describes a new algorithm to identify the current slopes and the derived current gradient vector of the freewheeling switching states of a VSI-driven 3phase synchronous machine. This is done by evaluation of the measured current slopes of the applied active switching states.

The proposed algorithm advances the art by enabling the use of the DACC-control scheme without the restriction of a minimum on-time of the zero switching state, which is up to now the state-of-the-art workaround in DACC implementations. As result, a significantly higher modulation index is possible, which means higher efficiency and reduced cost for the utilized inverter.

\section{REFERENCES}

[1] P. Cortes, M. Kazmierkowski, R. Kennel, D. Quevedo, and J. Rodriguez, "Predictive control in power electronics and drives," Industrial Electronics, IEEE Transactions on, vol. 55, no. 12 , pp. $4312-4324$, dec. 2008.

[2] A. Linder and R. Kennel, "Model predictive control for electrical drives," in Power Electronics Specialists Conference, 2005. PESC '05. IEEE 36th, june 2005, pp. 1793 -1799.

[3] T. Gemassmer, J. Richter, M. Schnarrenberger, and M. Braun, "High dynamic rotor oriented current control for permanent magnet synchronous machines with saturation characteristics," in PCIM Europe 2014, Nuremberg, Germany, 2014.

[4] J. Richter, T. Gemassmer, and M. Doppelbauer, "Predictive current control of saturated cross-coupled permanent magnet synchronous machines," in SPEEDAM 2014, Ischia (Italy), June 2014, 2014.

[5] J. Weigold and M. Braun, "Robust predictive dead-beat controller for buck converters," in Power Electronics and Motion Control Conference, 2006. EPE-PEMC 2006. 12th International, Aug 2006, pp. 951-956.

[6] F. Becker, H. Ennadifi, and M. Braun, "Straightforward current control - one step controller based on current slope detection," in EPE ECCE-EUROPE 2011 Birmingham, 2011.

[7] F. Becker, "Ein neues adaptives verfahren zur hochdynamischen stromregelung," Ph.D. dissertation, Elektrotechnisches Institut, Karlsruher Institut für Technologie (KIT), 2011. 

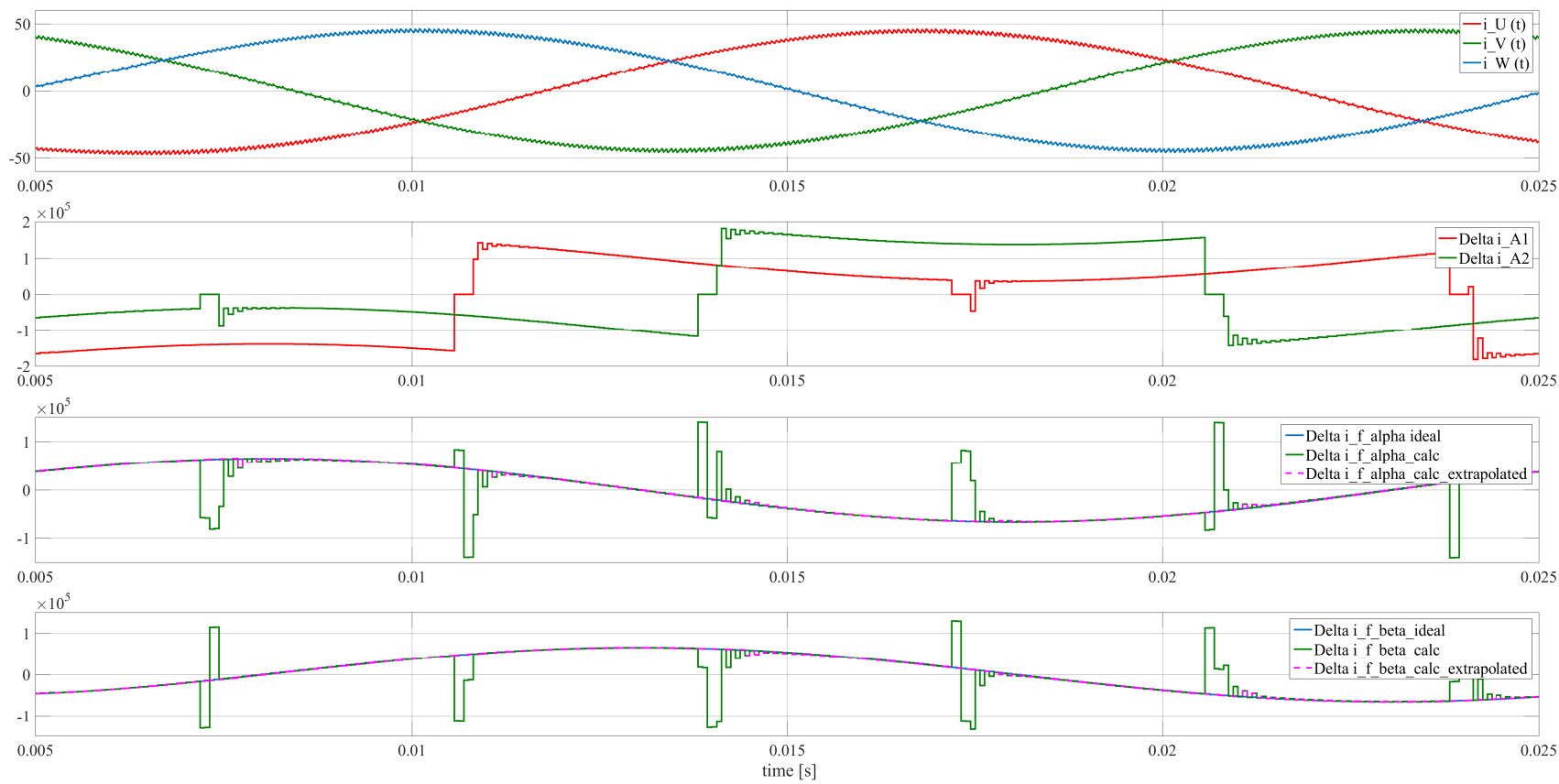

Figure 5. Timeline over one electrical period of the machine currents (top chart). The current gradient vectors of the active switching states are drawn in the second chart. In the two bottom charts, the ideal value of $\Delta \mathbf{i}_{\mathrm{f}}$ is compared to the calculated ones. The one without extrapolation delivers significantly false results, when one of the active switching states current slopes are not measurable. However, the extrapolated value is almost identical to the ideal value.

[8] A. Liske, P. Hofmeier, and M. Braun, "Extended straightforward current control for permanent magnet synchronous machines," in Power Electronics and Applications (EPE), 2013 15th European Conference on, 2013, pp. 1-10.

[9] A. Liske and M. Braun, "Direct adaptive current control - a universal current control scheme for electrical machines," in The 40th Annual Conference of the IEEE Industrial Electronics Society (IECON), 2014, 2014, pp. 1-7.

[10] J. Holtz, "Pulsewidth modulation for electronic power conversion," Proceedings of the IEEE, vol. 82, no. 8, pp. 1194-1214, Aug 1994.

[11] J. Weigold and M. Braun, "Predictive current conntrol using identificationn of current ripple," IEEE Transactions onn Industrial Electronnics, vol. 55, no. 2, pp. 4346-4353, 2008.

[12] P.-H. Liu, Y. Yan, F. Lee, and P. Mattavelli, "External ramp autotuning for current mode control of switching converters," in Applied Power Electronics Conference and Exposition (APEC), 2013 Twenty-Eighth Annual IEEE, March 2013, pp. 276-280.

[13] A. Liske, F. Stamer, and M. Braun, "Easy current slope detection for low cost implementation of the direct adaptive current control for dc-dc-converters," in 2015 IEEE Energy Conversion Congress and Exposition (ECCE), Sept 2015, pp. 180-186. 